\title{
ON THE NEIGHBOURHOODS OF STRONGLY CONVEX FUNCTIONS
}

\author{
R. PARVATHAM AND MILLICENT PREMABAI
}

\begin{abstract}
In this paper neighbourhoods of strongly convex and strongly starlike function are determined.
\end{abstract}

\section{Introduction}

Let $H(E)$ denote the class of all functions $f$ holomorphic in the open unit disc $E$ in $\mathbb{C}$ and $A$ be the class of all functions $f \in H(E)$ with the normalizations $f(0)=0=f^{\prime}(0)-1$. Any $f \in A$ has the Taylor's expansion $f(z)=z+a_{2} z^{2}+\ldots$ in $E$. The convolution or Hadamard product of $f(z)=z+\sum_{k=2}^{\infty} a_{k} z^{k}$ and $g(z)=z+\sum_{k=2}^{\infty} b_{k} z^{k}$ is defined as $(f * g)(z)=z+\sum_{k=2}^{\infty} a_{k} b_{k} z^{k}$. Clearly $f(z)=f(z) * f_{1}(z)$ and $z f^{\prime}(z)=f(z) * f_{2}(z)$ where

$$
f_{1}(z)=\frac{z}{1-z} \quad \text { and } \quad f_{2}(z)=\frac{z}{(1-z)^{2}}
$$

In this paper let us investigate the neighbourhoods of functions which are Strongly Starlike or Strongly Convex. These functions were introduced and discussed by D. A. Brannan and W. E. Kirwan [1] and also by J. Stankiewicz [5] and [6].

Definition 1. A function $f \in A$ is said to be Strongly Starlike of order $\alpha$, $0<\alpha \leq 1$ in $E$ if for all $z \in E,\left|\arg \frac{z f^{\prime}(z)}{f(z)}\right|<\frac{\alpha \pi}{2}$. The set of all such functions is denoted by $S^{*}(\alpha)$.

Clearly $S^{*}(1)=S^{*}$ (the class of all starlike functions).

$f \in S^{*}(\alpha)$ means that the image of $E$ under $\frac{z f^{\prime}(z)}{f(z)}$ lies in the region

$$
\Omega=\left\{z \in \mathbb{C}:|\arg z|<\frac{\alpha \pi}{2}, 0<\alpha \leq 1\right\}
$$

Received August 22, 1994.

1991 Mathematics Subject Classification. 30C45

Key words and phrases. Stronly starlike, strongly convex function. 
Equivalently $\frac{z f^{\prime}(z)}{f(z)} \neq t e^{ \pm i \alpha \pi / 2}, t \in \mathbb{R}^{+}$

Definition 2. Any function $f \in A$ is said to be Strongly Convex of order $\alpha$ in $E$ if for all $z \in E$,

$$
\left|\arg \left(1+\frac{z f^{\prime \prime}(z)}{f^{\prime}(z)}\right)\right|<\frac{\alpha \pi}{2}, \quad 0<\alpha \leq 1 .
$$

Let $K(\alpha)$ be the class of all strongly convex functions of order $\alpha$.

Note. (i) $K(1)=K$ - the class of all convex functions.

(ii) $f \in K(\alpha) \Longleftrightarrow z f^{\prime}(z) \in S^{*}(\alpha)$.

First let us state two lemmas (without proofs) which we need to establish our results in the sequel.

Lemma A. [2] Let $\beta^{\prime}, \tau^{\prime} \in \mathbb{C}, h \in H(E)$ be convex univalent in $E$ with $h(0)=1$ and $\operatorname{Re}\left(\beta^{\prime} h(z)+\tau^{\prime}\right)>0, z \in E$ and let $p(z)=1+p_{1} z+\cdots \in H(E)$. Then

$$
p(z)+\frac{z p^{\prime}(z)}{\beta p(z)+\tau} \prec h(z) \Longrightarrow p(z) \prec h(z)
$$

where the symbol $\prec$ denotes subordination.

Lemma B. [4] If $\phi$ is a convex univalent function with $\phi(0)=0=\phi^{\prime}(0)-1$ in the unit disk $E$ and $g$ is starlike univalent in $E$, then for each analytic function $F$ in $E$, the image of $E$ under $\frac{(\phi * F g)(z)}{(\phi * g)(z)}$ is a subset of the convex hull of $F(E)$.

First let us establish an inclusion relation.

Theorem 1. Let $f \in K(\alpha)$. Then $f \in S^{*}(\alpha)$.

Proof. Let $p(z)=\frac{z f^{\prime}(z)}{f(z)}$. Then since $f \in K(\alpha), p(z)+\frac{z p^{\prime}(z)}{p(z)}=1+\frac{z f^{\prime \prime}(z)}{f^{\prime}(z)} \subset \Omega$, defined in (1). Since $\Omega$ is a convex domain, an application of Lemma A gives $\left[\frac{z f^{\prime}(z)}{f(z)}\right]_{z \in E}=$ $p(z)_{z \in E} \subset \Omega$, which shows that $f \in S^{*}(\alpha)$.

Definition 3. For $f \in S^{*}(\alpha)$ define $S^{* \prime}(\alpha)$ as a class of all functions

$$
h(z)=\frac{f_{2}(z)-t e^{ \pm i \alpha \pi / 2} f_{1}(z)}{1-t e^{ \pm i \alpha \pi / 2}}
$$

$t \in \mathbb{R}^{+}$where $f_{1}(z)=\frac{z}{1-z}$ and $f_{2}(z)=\frac{z}{(1-z)^{2}}$.

Now let us give a characterization for a function $f \in A$ to be in $S^{*}(\alpha)$ be means of convolution.

Theorem 2. $f \in S^{*}(\alpha)$ if and only if $\frac{(f * H)(z)}{z} \neq 0, z \in E$ and for all $H(z) \in S^{* \prime}(\alpha)$. 
Proof. First let us assume that $\frac{(f * H)(z)}{z} \neq 0$ for all $H \in S^{* \prime}(\alpha)$ and $z \in E$. Hence

$$
\frac{(f * H)(z)}{z}=\frac{f(z) * \frac{z}{(1-z)^{2}}-t e^{ \pm i \alpha \pi / 2} f(z) * \frac{z}{1-z}}{z\left(1-t e^{ \pm i \alpha \pi / 2}\right)}, \quad t \in \mathbb{R}^{+} \neq 0 .
$$

Equivalently $\frac{z f^{\prime}(z)}{f(z)}+t e^{ \pm i \alpha \pi / 2}, t \in \mathbb{R}^{+}$. As $t \in \mathbb{R}^{+}, t e^{ \pm i \alpha \pi / 2}$ covers the straight line $\arg w= \pm \alpha \pi / 2$ and $\frac{z f^{\prime}(z)}{f(z)}=1$ at $z=0$, Hence

$$
\frac{z f^{\prime}(z)}{f(z)} \in \Omega=\left[z \in \mathbb{C}:\left|\arg \frac{z f^{\prime}(z)}{f(z)}\right|<\frac{\alpha \pi}{2}\right] \text { or } f \in S^{*}(\alpha)
$$

Conversely let $f \in S^{*}(\alpha)$. Then

$$
\frac{z f^{\prime}(z)}{f(z)} \neq t e^{ \pm i \alpha \pi / 2}, t \in \mathbb{R}^{+}
$$

Now

$$
\begin{aligned}
\frac{(f * H)(z)}{z} & =\frac{f(z) * f_{2}(z)-t e^{ \pm i \alpha \pi / 2} f_{1}(z)}{z\left(1-t e^{ \pm i \alpha \pi / 2}\right)} \\
& =\frac{\frac{z f^{\prime}(z)}{f(z)}-t e^{ \pm i \alpha \pi / 2}}{\left(1-t e^{ \pm i \alpha \pi / 2}\right)}\left[\frac{f(z)}{z}\right]
\end{aligned}
$$

(2) gives $\frac{(f * H)(z)}{z} \neq 0$ in $E$ which completes the proof of the theorem.

The notion of $\delta$-neighbourhood was first introduced by St. Ruscheweyh [3].

Definition 4. For $\delta \geq 0$ the $\delta$-neighbourhood of $f(z)=z+\sum_{k=2}^{\infty} a_{k} z^{k} \in A$ is defined by

$$
N_{\delta}(f)=\left[g(z)=z+\sum_{k=2}^{\infty} b_{k} z^{k}: \sum_{k=2}^{\infty} k\left|a_{k}-b_{k}\right| \leq \delta\right]
$$

Lemma 1. Let $H(z)=z+\sum_{k=2}^{\infty} h_{n} z^{n} \in S^{* \prime}(\alpha)$. Then

$$
\left|h_{n}\right| \leq \frac{n}{\sin \frac{\alpha \pi}{2}} .
$$

Proof. Since $H(z) \in S^{* \prime}(\alpha)$, we have

$$
\begin{aligned}
H(z) & =\frac{1}{1-t e^{ \pm i \alpha \pi / 2}}\left[\frac{z}{(1-z)^{2}}-t e^{ \pm i \alpha \pi / 2} \frac{z}{1-z}\right] \\
& =z+\sum_{k=2}^{\infty} h_{n} z^{n} .
\end{aligned}
$$


Then comparing the coefficients on either sides we get,

$$
\begin{aligned}
\left|h_{n}\right| & =\left|\frac{n-t e^{ \pm i \alpha \pi / 2}}{1-t e^{ \pm i \alpha \pi / 2}}\right| . \text { Hence } \\
\left|h_{n}\right|^{2} & =\frac{(n-t \cos (\alpha \pi / 2))^{2}+t^{2} \sin ^{2}(\alpha \pi / 2)}{(1-t \cos (\alpha \pi / 2))^{2}+t^{2} \sin ^{2}(\alpha \pi / 2)} \\
& =\frac{n^{2}-2 n t \cos (\alpha \pi / 2)+t^{2}}{1-2 t \cos (\alpha \pi / 2)+t^{2}} \\
\left|h_{n}\right|^{2} & =1+\frac{n^{2}-1-2 t(n-1) \cos (\alpha \pi / 2)}{1-2 t \cos (\alpha \pi / 2)+t^{2}} \\
& \leq 1+\frac{n^{2}-1}{1-2 t \cos (\alpha \pi / 2)+t^{2}} \text { since } t \geq 0 ; \\
& \leq \max _{t}\left[1+\frac{n^{2}-1}{1-2 t \cos (\alpha \pi / 2)+t^{2}}\right] \\
& \leq 1+\frac{n^{2}-1}{\sin ^{2}(\alpha \pi / 2)}=\frac{n^{2}-\cos ^{2}(\alpha \pi / 2)}{\sin ^{2}(\alpha \pi / 2)} .
\end{aligned}
$$

Therefore

$$
\left|h_{n}\right| \leq \frac{\sqrt{n^{2}-\cos ^{2}(\alpha \pi / 2)}}{\sin (\alpha \pi / 2)}<\frac{n}{\sin (\alpha \pi / 2)}
$$

Lemma 2. For $f \in A$ and for every $\epsilon \in \mathbb{C}$ such that $|\epsilon|<\delta$, if $F_{\epsilon}(z)=$ $\frac{f(z)+\epsilon z}{1+\epsilon} \in S^{*}(\alpha)$ then for every $H \in S^{* \prime}(\alpha),\left|\frac{(f * H)(z)}{z}\right| \geq \delta, z \in E$.

Proof. Let $F_{\epsilon} \in S^{*}(\alpha)$. Then by Theorem $2, \frac{\left(F_{\epsilon} * H\right)(z)}{z} \neq 0$, for all $H \in S^{* \prime}(\alpha)$, $z \in E$. Equivalently

$$
\frac{(f * H)(z)+\epsilon z}{(1+\epsilon) z} \neq 0 \quad \text { in } E \text { or } \quad \frac{(f * H)(z)}{z} \neq-\epsilon
$$

which shows that $\left|\frac{(f * H)(z)}{z}\right| \geq \delta$.

Theorem 3. For $f \in A$ and $\epsilon \in \mathbb{C},|\epsilon|<\delta<1$ assume $F_{\epsilon}(z) \in S^{*}(\alpha)$. Then $N_{\delta \sin (\alpha \pi / 2)}(f) \subset S^{*}(\alpha)$. 
Proof. Let $H \in S^{* \prime}(\alpha)$ and $g(z)=z+\sum_{k=2}^{\infty} b_{k} z^{k}$ is in $N_{\delta^{\prime}}(f)$. Then

$$
\begin{aligned}
\left|\frac{(g * H)(z)}{z}\right| & =\left|\frac{(f * H)(z)}{z}+\frac{((g-f) * H)(z)}{z}\right| \\
& \geq\left|\frac{(f * H)(z)}{z}\right|-\left|\frac{(g-f)(z) * H(z)}{z}\right| \\
& \geq \delta-\left|\sum_{k=2}^{\infty} \frac{\left(b_{k}-a_{k}\right) h_{k} z^{k}}{z}\right| \quad \text { by Lemma } 2 ; \\
\text { thus } & \\
\left|\frac{(g * H)(z)}{z}\right| & \geq \delta-|z| \sum_{k=2}^{\infty}\left|h_{k}\right|\left|b_{k}-a_{k}\right| ; \\
& >\delta-\frac{1}{\sin (\alpha \pi / 2)} \sum_{k=2}^{\infty}\left|b_{k}-a_{k}\right| k \text { by Lemma } 1 \\
& >\delta-\frac{\delta^{\prime}}{\sin (\alpha \pi / 2)}=0 \text { for } \delta^{\prime}=\delta \sin (\alpha \pi / 2)
\end{aligned}
$$

Thus $\frac{(g * H)(z)}{z} \neq 0$ in $E$ for all $H \in S^{* \prime}(\alpha)$ which means by Theorem $2, g \in S^{*}(\alpha)$; in otherwords $N_{\delta} \sin (\alpha \pi / 2)(f) \subset S^{*}(\alpha)$.

Next let us show that the class $S^{*}(\alpha)$ is closed under convolution with functions $f$ which are convex univalent in $E$, that is $(f * g)(z) \in S^{*}(\alpha)$ whenever $f \in K$ and $g \in S^{*}(\alpha)$.

Theorem 4. Let $f(z) \in K, g(z) \in S^{*}(\alpha)$. Then $(f * g)(z) \in S^{*}(\alpha)$.

Proof. Since $g \in S^{*}(\alpha) \subset S^{*}$ the class of starlike functions and $f \in K$ and $\Omega$ defined by (1) is a convex domain, an application of Lemma $B$ gives

$$
\begin{aligned}
\frac{z(f * g)^{\prime}(z)}{(f * g)(z)} & =\frac{f *\left[\frac{z g^{\prime}(z)}{g(z)}\right] g(z)}{(f * g)(z)} \\
& \subset \bar{c}_{0} \frac{z g^{\prime}(z)}{g^{\prime}(z)}=\Omega, \quad z \in E
\end{aligned}
$$

This shows that $(f * g)(z) \in S^{*}(\alpha)$.

Theorem 5. If $f \in K(\alpha)$, then $\frac{f(z)+\epsilon z}{1+\epsilon} \in S^{*}(\alpha)$ for $|\epsilon|<\frac{1}{4}$. 
Proof. Let $f(z)=z+\sum_{n=2}^{\infty} a_{n} z^{n}$. Then

$$
\begin{aligned}
\frac{f(z)+\epsilon z}{1+\epsilon} & =\frac{z(1+\epsilon)+\sum_{n=2}^{\infty} a_{n} z^{n}}{1+\epsilon} \\
& =\frac{f(z) *\left[z(1+\epsilon)+\sum_{n=2}^{\infty} z^{n}\right]}{1+\epsilon} \\
& =f(z) * \frac{\left[z-\frac{\epsilon}{1+\epsilon} z^{2}\right]}{1-z}=f(z) * h(z)
\end{aligned}
$$

where $h(z)=\frac{\left[z-\frac{\epsilon}{1+\epsilon} z^{2}\right]}{1-z}$. Now

$$
\frac{z h^{\prime}(z)}{h(z)}=\frac{\left[z-\frac{2 \epsilon}{1+\epsilon} z^{2}\right]}{\left[z-\frac{\epsilon}{1+\epsilon} z^{2}\right]}+\frac{z}{1-z}=\frac{-\rho z}{1-\rho z}+\frac{1}{1-z}
$$

where $\rho=\frac{\epsilon}{1+\epsilon}$. Hence $|\rho|<\frac{\epsilon}{1-|\epsilon|}<\frac{1}{3}$ gives $|\epsilon|<\frac{1}{4}$. Thus $\operatorname{Re}\left[\frac{z h^{\prime}(z)}{h(z)}\right] \geq \frac{1-2|\rho||z|-|\rho||z|^{2}}{(1-|\rho||z|)(1+|z|)}>0$ if $\left.|\rho|<|z|^{2}+2|z|\right)-1<0$. This inequality holds for all $\rho<1 / 3$. and $|z|<1$, which is true for $|\epsilon|<1 / 4$. Therefore $h$ is starlike in the unit disk and so $\int_{0}^{z} \frac{h(t)}{t} d t$ is convex.

But $h(z) * \log \left[\frac{1}{1-z}\right]=\int_{0}^{z} \frac{h(t)}{t} d t$ and so $h(z) * \log \left[\frac{1}{1-z}\right]$ is convex in $E$ and

$$
\begin{aligned}
(f * h)(z)=(h * f)(z) & =h(z) *\left[z f^{\prime}(z) * \log \left[\frac{1}{1-z}\right]\right] \\
& =z f^{\prime}(z) *\left[\left(h(z) * \log \left[\frac{1}{1-z}\right]\right]\right.
\end{aligned}
$$

$f(z) \in K(\alpha) \Longrightarrow z f^{\prime}(z) \in S^{*}(\alpha)$ and $\left.h(z) * \log \left[\frac{1}{1-z}\right]\right] \in K$. Now by Theorem 4 $h(z) *\left[z f^{\prime}(z) * \log \left[\frac{1}{1-z}\right]\right]$ is in $S^{*}(\alpha)$. Thus $(f * h)(z)=\frac{f(z)+\epsilon z}{1+\epsilon} \in S^{*}(\alpha)$ for $|\epsilon|<1 / 4$.

Theorem 6. Let $f \in K(\alpha)$. Then $N_{1 / 4} \sin (\alpha \pi / 2)(f) \subset S^{*}(\alpha)$.

Proof. Let $f \in K(\alpha)$. Then from Theorem 5 we have $\frac{f(z)+\epsilon}{1+\epsilon} \in S^{*}(\alpha)$ for $|\epsilon|<1 / 4$. Then an application of Theorem 3 gives $N_{1 / 4} \sin (\alpha \pi / 2)(f) \subset S^{*}(\alpha)$.

When $\alpha=1$ we get a result of St. Ruscheweyh [3] as a special case.

\section{Refercence}

[1] D. A. Brannan and W. E. Kirwan, "On some classes of bounded univalent functions," J. London Math. Soc. 1(2)(1969), 431-443.

[2] P. Eenigenburg, S. S. Miller, P. T. Mocanu and M. O. Reade, "On a Briot-Bouquet Differential Subordination," General Inequalities 3, Birkhauser Verlag-Basel, 339-348. 
[3] St. Ruscheweyh, "Neighbourhoods of univalent functions," Proc. Amer. Math. Soc., 81(1981), 521527.

[4] St. Ruscheweyh and T. Sheil-Small, "Hadamard Products of Schilicht functions and the PolyaSchoenberg Conjecture," Comment. Math. Helvi, 48(1973), 119-135.

[5] J. Stankiewicz, "Quelques problems extremanx dans des classes de functions $\alpha$-angulairement etoilees," Ann. Univ. M. Curie-Sklodowska, Section A, 20(1966), 59-75.

[6] J. Stankiewicz, "Some remarks concerning Starlike functions," Bull. Acad. Polon. Sci. Ser. Sci. Math., 18(1970), 143-146.

Ramanujan Institute, University of Madras, Madras-600 005, India.

Anna Adarsh College, (Affliated to the University of Madras), Anna Nagar, Madras-600 040, India. 